\title{
A Machine Learning Recommender System Based on Collaborative Filtering Using Gaussian Mixture Model Clustering
}

\author{
Delshad Fakoor ${ }^{1}$, Vafa Maihami ${ }^{1}$, and Reza Maihami ${ }^{2}$ \\ ${ }^{1}$ Islamic Azad University Sanandaj Branch \\ ${ }^{2}$ Our Lady of the Lake University of San Antonio
}

December 26, 2020

\begin{abstract}
Changing and moving toward online shopping has made it necessary to customize customers' needs and provide them more selective options. The buyers search the products' features before deciding to purchase items. The recommender systems facilitate the searching task for customers via narrowing down the search space within the specific products that align the customer needs. Clustering, as a typical machine learning approach, is applied in recommender systems. As an information filtering method, a recommender system clusters user's data to indicate the required factors for more accurate predictions by calculating the similarity between members of a cluster. In this study, using the Gaussian mixture model clustering and considering the scores distance and the value of scores in the Pearson correlation coefficient, a new method is introduced for predicting scores in machine learning recommender systems. To study the proposed method's performance, a Movie Lens data set is evaluated, and the results are compared to some other recommender systems, including the Pearson correlation coefficients similarity criteria, K-means, and fuzzy C-means algorithms. The simulation results indicate that our method has less error than others by increasing the number of neighbors. The results also illustrate that when the number of users increases, the proposed method's accuracy will increase. The reason is that the Gaussian mixture clustering chooses similar users and considers the scores distance in choosing similar neighbors to the active user.
\end{abstract}

\section{Hosted file}

Manuscript.pdf available at https://authorea.com/users/385213/articles/500710-a-machinelearning-recommender-system-based-on-collaborative-filtering-using-gaussian-mixturemodel-clustering 


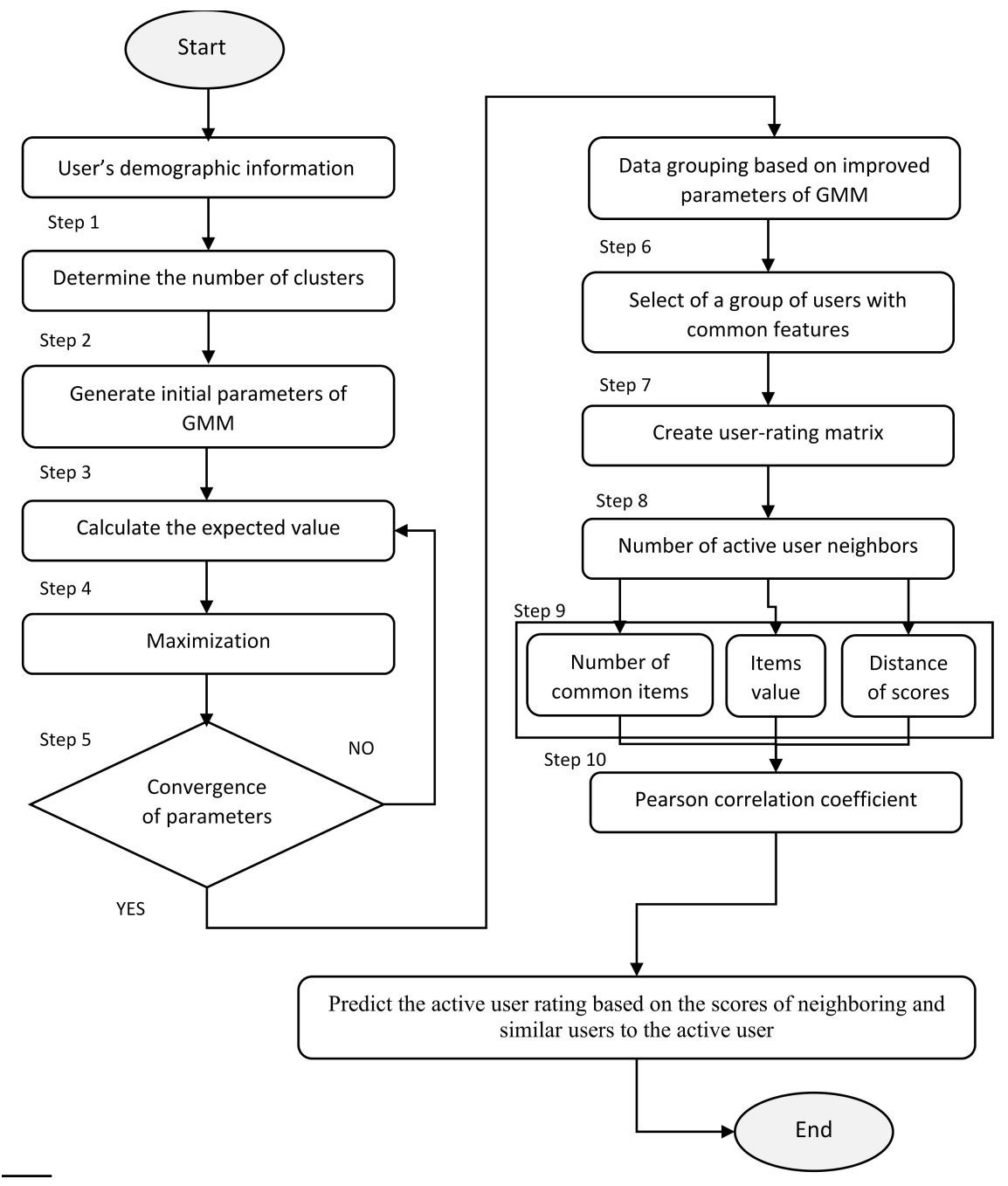



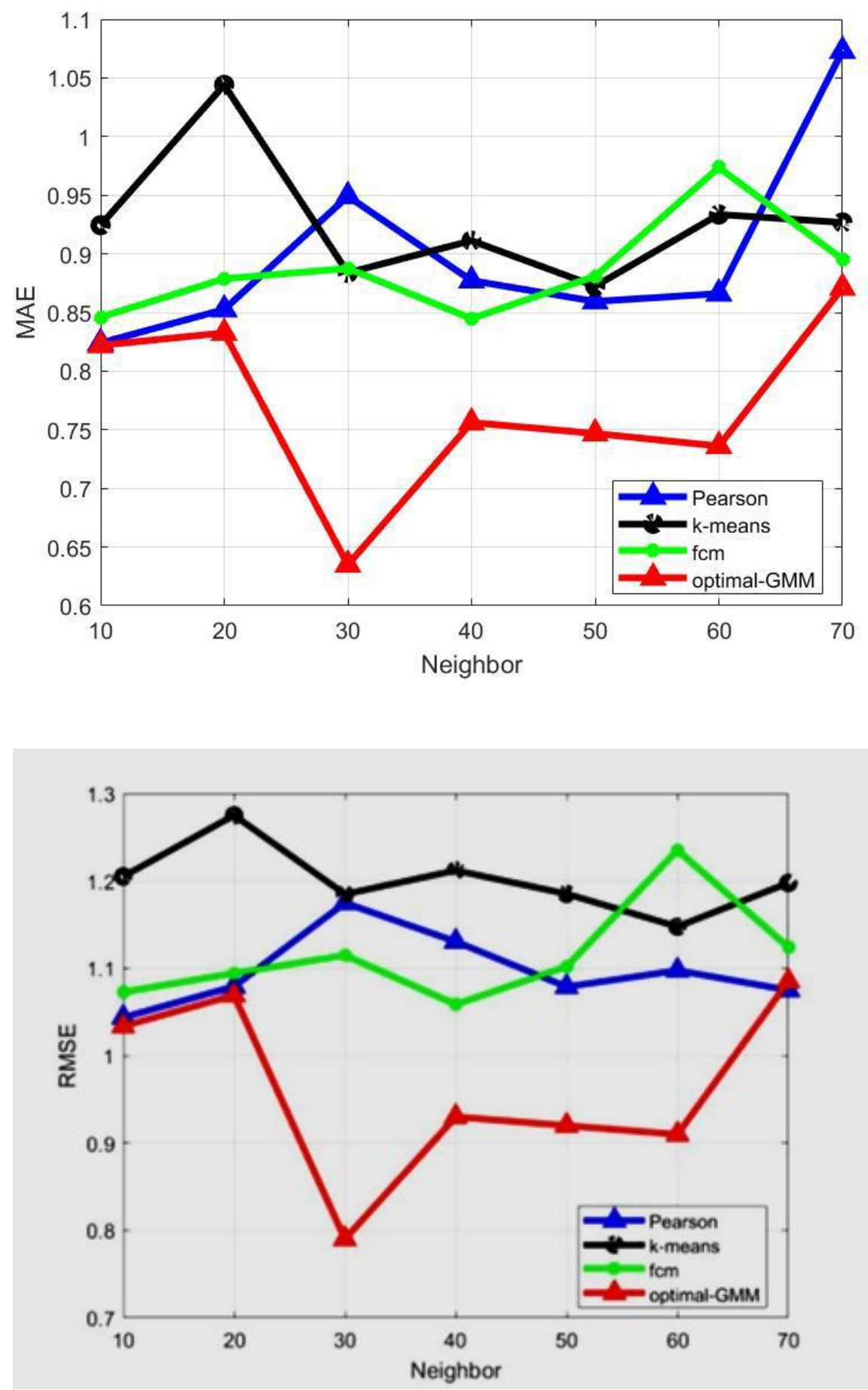